\title{
1 Microbial metabolites mediate bumble bee attraction and feeding
}

3 Robert N. Schaeffer ${ }^{1,2,4, \dagger}$, Caitlin C. Rering ${ }^{3, \dagger}$, Isabelle Maalouf ${ }^{2}$, John J. Beck ${ }^{3}$, Rachel L.

4 Vannette ${ }^{2}$

$6 \quad{ }^{1}$ Department of Entomology, Washington State University, Pullman, WA 99164

$7 \quad{ }^{2}$ Department of Entomology and Nematology, University of California Davis, Davis, CA 95616

$8{ }^{3}$ Chemistry Research Unit, Center for Medical, Agricultural and Veterinary Entomology, 32608

$12{ }^{4}$ Corresponding author and current address: Department of Biology, Utah State University,

13 Logan, UT 84321, robert.schaeffer@usu.edu

$14{ }^{\dagger}$ Equally-contributing authors

\section{Abstract}

Animals such as bumble bees use chemosensory cues to localize and evaluate essential

18 resources. Increasingly, it is recognized that microbes can alter the quality of foraged resources

19 and produce metabolites that act as foraging cues. The distinct nature of these sensory cues

20 however and their use in animal foraging remain poorly understood. Here, we test the hypothesis

21 that species of nectar-inhabiting microbes differentially influence pollinator attraction and

22 feeding via microbial metabolites in nectar. We examined electrophysiological potential of

23 bumble bee antennae to respond to volatile microbial metabolites, followed by behavioral

24 responses using choice assays. We assessed gustatory responses through both no-choice and

25 choice feeding assays. Antennae responded to some microbial volatiles, and bees chose Asaia

26 bacterial solutions compared to Metschnikowia yeast based on volatiles alone. However, $B$.

27 impatiens consumed significantly more Metschnikowia-inoculated nectar, suggesting distinct

28 roles for volatile and non-volatile microbial metabolites in mediating feeding decisions, with

29 potential to affect associative learning and future foraging. Our results suggest that microbial

30 metabolites may represent non-reinforcing cues with potential consequences for forager learning,

31 economics and floral host reproduction. 
33 Keywords: Asaia, bumble bee, Metschnikowia reukaufii, nectar microbes, pollination, volatile

34 organic compounds

Introduction

To successfully persist in a chemosensory environment, animals must receive and interpret cues and signals of ecologically-important information, such as the quantity and quality of resources potentially available to them [1]. This is especially true of pollinators such as bumble bees, which integrate multi-modal signals, including form, color, and scent, to accurately identify rewarding flowers [2]. Like other food resources, flowers host varied microbial species and communities [3,4], which produce metabolites that may act as cues of resource availability and quality, with consequences for pollinator foraging [5,6]. Indeed, insect pollinators are highly sensitive to shifts in volatile abundance and identity [7-9], with scents being known to influence learned foraging preferences [10]. However, the role of volatile and non-volatile microbial metabolites in mediating pollinator attraction and foraging decisions still remains largely unclear.

In standing crop nectar, bacteria and fungi colonize between $20-70 \%$ of individual flowers, attain densities exceeding $10^{5}$ and $10^{7}$ cells $/ \mu \mathrm{L}$ respectively $[3,4]$ and metabolize sugars and amino acids [5,11], affecting pollinator foraging and plant reproduction [5,12,13]. Intense competition between microbes in nectar often results in flowers that are dominated by either yeast or bacteria [14]. Yeasts and bacteria differ in volatile composition and acceptance to pollinators [6], but also differentially influence non-volatile nectar traits (Vannette \& Fukami 2018) and shift pollinator perceptions of nectar quality [15]. Predicting microbial effects on

54 pollinator foraging and behavior requires examining responses to olfactory (headspace volatiles)

55 and gustatory (dissolved chemicals) cues.

Here, we test the hypothesis that yeast and bacteria differentially influence bumblebee attraction and feeding. Bumble bees (Bombus impatiens) are an ideal system, due to their close ecological relationships with yeasts $[16,17]$ and bacteria $[18,19]$. We examined antennal responses to microbial metabolites using electroanntenographic (EAG) bioassays, bee choice using olfactometer (Y-tube) bioassays, and gustatory preferences using choice and no-choice

61 feeding assays. We found bumble bees show distinct responses to volatile vs gustatory microbial 62 cues to inform foraging decisions, indicating the potential for associative learning, where bumble 
63 bees may adjust behavioral responses to volatile blends after exposure to gustatory microbial

64 cues.

Materials and methods

Study system

We used three colonies of the generalist bumble bee Bombus impatiens (Koppert Biological Systems, Inc.; Howell, MI, USA) and strains of the nectar-inhabiting yeast Metschnikowia reukaufii (Metschnikowiaceae; GenBank ID: MF319536) and bacteria Asaia astilbes (Acetobacteraceae; GenBank ID: KC677740). Both M. reukaufii, and A. astibles are

72 commonly isolated from floral nectar [20] and pollinators (Good et al. 2014), but differentially

73 influence nectar chemistry and scent [21].

Experiment 1: Electroantennographic bioassay

We examined antennal response ( $\mathrm{n}=6$ /metabolite) to volatiles produced by Metschnikowia and Asaia (Table 1) by puffing each metabolite $(0.4 \mu \mathrm{mol})$ over excised $B$. impatiens antennae. Antennal responses were recorded and corrected by responses to blanks and positive control stimuli ( $0.4 \mu \mathrm{mol}$ geraniol), see electronic supplementary material S1 Methods.

To assess whether bumble bees exhibit innate preferences when exposed to volatile microbial metabolites, we used an olfactometer assay (Y-tube; Fig. S1) under red light. Naïve bumble bees housed at the University of California Davis were starved for 6 hours, then released individually into the Y-tube. For each bee, initial choice and the time spent in each arm was recorded, and the assay was repeated twice for each bee, with treatment direction reversed. These bees were both fed and treated similarly to those used for the EAG assays and a total of 32 bees were tested in this assay from two source colonies. For details, see electronic supplementary

\section{Experiment 3: Gustatory responses of bumble bees to nectar-inhabiting microbes}

To assess gustatory responses of bumble bees ( $n=42$ bees from two colonies) to nectar

93 colonized by microbial taxa, we used both no-choice and choice feeding assays. For the no- 
94 choice assay, bees were housed in individual vials with modified lids that accommodated a

95 feeding apparatus (Fig. S2). Vials were filled with $1 \mathrm{~mL}$ of either Asaia- or Metschnikowia-

96 treated nectar, weighed, and bees were allowed to feed for $24 \mathrm{hr}$, after which tubes were re-

97 weighed to determine consumption. For details, see electronic supplementary material S1

98 Methods.

\section{Experiment 4: Effects of volatile and gustatory microbial cues on associative learning}

Because bees exhibited marked differences in response to volatile and gustatory microbial cues (see Results below), we also assessed how exposure to gustatory cues influenced

103 bee preference for volatiles ( $\mathrm{n}=24$ bees from two colonies). Individual foragers were subjected to

104 the olfactometer assay (above), then a gustatory choice assay where individual bees were housed

105 in a feeding chamber, consisting of $\sim 9 \mathrm{~cm}$ of perforated tubing, with feeding vials on either end

106 of the chamber (Fig. S3) for $24 \mathrm{hr}$. Vials were weighed to determine nectar consumption. Bees

107 were then subjected to a second olfactometer assay.

Statistical analyses

110 To assess which compounds were detected by bumble bees (Experiment 1), we used

$111 t$-tests with false discovery rate correction to examine if normalized EAG responses were

112 significantly different from zero (i.e., no detectable response). To determine if bee preference

113 differed between microbes, data from Experiment 2 were analyzed using a binomial test for first

114 choice. A linear mixed-effect (LME) model [22] was used for time spent in each arm, with

115 microbial treatment as a fixed effect, and bee individual as a random effect. For gustatory cues

116 (Experiment 3), we used a $t$-test to assess how nectar consumption was affected by the nectar

117 treatment. For Experiment 4, we fit a LME model with proportion of time spent in olfactometer

118 arms as the response variable, nectar treatment, choice test order, and their interaction as fixed

119 effects, and bee individual as a random effect. Bumble bee feeding responses were also analyzed

120 with a LME model, with amount consumed as the response variable, nectar treatment as a fixed

121 effect, and bee individual as a random effect. All analyses were performed in R (v. 3.5.2) [23].

122

\section{Results}


Bumble bee antennae responded to a subset (4/20) of volatile metabolites tested through

125 EAG (Experiment 1; Table 1) at $0.4 \mu \mathrm{mol}$, including 1-hexanol, 2-ethyl-1-hexanol, 2-

126 phenylethanol, and 3-methylbutyl acetate (i.e., isoamyl acetate, isopentyl acetate). The alcohol 2-

127 ethyl-1-hexanol elicited the strongest EAG depolarization response, surpassing that of the

128 positive control $(0.4 \mu \mathrm{mol}$ geraniol $)$.

129 Volatile blends emitted by nectar-inhabiting microbes also influenced bee behavior.

130 Naïve bees on average spent $\sim$ two-thirds of their time in Y-tube arms containing Asaia-produced

131 volatiles (Figure $1 \mathrm{~A} ; F_{1,64}=21.52, P<0.0001$ ), although no difference was found for first choice

$132(P=0.67)$. In contrast, bees consumed approximately 50\% less Asaia-conditioned nectar than

133 Metschnikowia nectar (Figure $1 \mathrm{~B} ; t_{29.5}=-2.70, P=0.011$ ) in a no-choice assay (Experiment 3 ).

134 Mirroring earlier results, bumble bees across both choice tests performed in Experiment 4

135 spent $\sim 15 \%$ more of their time in the Y-tube arm assigned to Asaia compared to that of

136 Metschnikowia $\left(F_{1,163}=9.09, P=0.003\right)$. During the feeding assay, bees again consumed on

137 average nearly double the amount of Metschnikowia-conditioned nectar compared to Asaia

$138\left(F_{1,46}=12.29, P=0.001\right)$. After experiencing gustatory cues in the feeding assay, bees reduced the

139 frequency with which they chose the Asaia volatile blend, increasing both the proportion of "no

140 choice' and that for the yeast arm, as well as the amount of time spent (albeit not significant) in

141 the Metschnikowia arm of the olfactometer.

\section{Discussion}

144 Microbes commonly inhabit food resources, contributing both volatile and non-volatile

145 metabolites that can function to inform foragers as to their location and quality. We found

146 distinct effects of olfactory vs. gustatory cues produced by two common, nectar-inhabiting

147 microbes on bumble bee behavior and nectar consumption. The difference in bee response to

148 olfactory vs. gustatory cues suggests that these cues are not reinforcing, which may complicate

149 pollinator foraging and learning based on microbial metabolites [24]. Further, bee preference for

150 Asaia volatiles decreased after exposure to gustatory cues and feeding, suggesting behavior

151 modification. We suspect that acetic acid produced by Asaia (but not Metschnikowia), although

152 not detectable in our volatile screening, may be aversive to bees. In natural systems, bees likely

153 develop associations between microbial chemosensory cues through repeated exposure to the

154 scent and taste of yeast or bacterial-colonized nectar. However, our findings, and recent 
experimental results [24], suggest that microbial signals may be more difficult to learn than other sensory combinations. Such difficulties may manifest to affect learned preferences, floral constancy and the quantity and quality of benefits exchanged in these mutualistic interactions. Collectively, our results indicate that volatile and non-volatile microbial metabolites have significant potential to shape interspecific, plant-pollinator signaling. In remains to be determined whether pollinators benefit from microbial-derived cues can translate to improved foraging efficiency, or whether such cues may be more exploitative, and benefit microbes that rely upon pollinator dispersal to reach new floral habitats [25]. Such outcomes may hinge on both the identity and density of the microbial species encountered, where varied immigration histories can give rise to divergent microbial communities both within flowers of a host and among other species. Our results demonstrate that future investigations on the evolutionary ecology of floral signaling should consider multiple ways in which microbes influence host phenotype and the innate and learned response of pollinators.

Authors' contributions. R.S., C.R., J.B., and R.V. conceived the study. R.S., C.R., and I.M. collected data, while R.S. and C.R. performed statistical analyses and drafted the manuscript. All authors contributed to manuscript editing, gave final approval for publication, and agree to be

172 held accountable for the worked performed therein.

\section{Competing interests.}

175 The authors have no competing interests.

\section{Funding.}

178 This research was supported by UC Davis and Hatch funds awarded to RV, USDA-ARS

179 Research Project 6036-22000-028 (JB and CR), and 2016 ARS Administrator Research

180 Associate program (CR). RS acknowledges support from a USDA NIFA Education and Literacy

181 Initiative Postdoctoral Fellowship (2017-67012-26104).

\section{Acknowledgements.}

184 We thank M. Handy, A. Khan, I. Munkres, H. Pathak, and M. Anderson for laboratory

185 assistance, and B. Forguson for greenhouse assistance. 


\section{References}

187 1. Carthey AJR, Gillings MR, Blumstein DT. 2018 The extended genotype: Microbially mediated olfactory communication. Trends Ecol. \& Evol. 33, 885-894. (doi:10.1016/j.tree.2018.08.010)

2. Schiestl FP, Johnson SD. 2013 Pollinator-mediated evolution of floral signals. Trends Ecol.

3. Herrera CM, de Vega C, Canto A, Pozo MI. 2009 Yeasts in floral nectar: a quantitative survey. Ann. Bot. 103, 1415-1423. (doi:10.1093/aob/mcp026)

4. Fridman S, Izhaki I, Gerchman Y, Halpern M. 2012 Bacterial communities in floral nectar. Environ. Micro. Rep. 4, 97-104. (doi:10.1111/j.1758-2229.2011.00309.x)

5. Vannette RL, Gauthier M-PL., Fukami T. 2013 Nectar bacteria, but not yeast, weaken a plantpollinator mutualism. Proc. R. Soc. B 280, 20122601. (doi:10.1098/rspb.2012.2601)

6. Rering CC, Beck JJ, Hall GW, McCartney MM, Vannette RL. 2018 Nectar-inhabiting microorganisms influence nectar volatile composition and attractiveness to a generalist pollinator. New Phyt. 220, 750-759. (doi:10.1111/nph.14809)

7. Dobson HEM. 2006 Relationship between Floral Fragrance Composition and Type of Pollinator. Biology of Floral Scent. (doi:10.1201/9781420004007-12)

8. Pichersky E, Gershenzon J. 2002 The formation and function of plant volatiles: perfumes for pollinator attraction and defense. Current Opinion in Plant Biology 5, 237-243.

205 (doi:10.1016/S1369-5266(02)00251-0)

9. Galen C, Kaczorowski R, Todd SL, Geib J, Raguso RA. 2011 Dosage-dependent impacts of a floral volatile compound on pollinators, larcenists, and the potential for floral evolution in the alpine skypilot Polemonium viscosum. Am. Nat. 177, 258-272. (doi:10.1086/657993)

10. Wright GA, Schiestl FP. 2009 The evolution of floral scent: the influence of olfactory learning by insect pollinators on the honest signalling of floral rewards. Funct. Ecol. 23, 841-851. (doi:10.1111/j.1365-2435.2009.01627.x)

11. Schaeffer RN, Vannette RL, Irwin RE. 2015 Nectar yeasts in Delphinium nuttallianum (Ranunculaceae) and their effects on nectar quality. Fung. Ecol. 18, 100-106. (doi:10.1016/j.funeco.2015.09.010)

12. Herrera CM, Pozo MI, Medrano M. 2013 Yeasts in nectar of an early-blooming herb: sought by bumble bees, detrimental to plant fecundity. Ecology 94, 273-279. (doi:10.1890/120595.1)

13. Schaeffer RN, Irwin RE. 2014 Yeasts in nectar enhance male fitness in a montane perennial herb. Ecology 95, 1792-1798. (doi:10.1890/13-1740.1) 
14. Toju H, Vannette RL, Gauthier M-PL, Dhami MK, Fukami T. 2018 Priority effects can persist across floral generations in nectar microbial metacommunities. Oikos 127, 345-352. (doi:10.1111/oik.04243)

15. Vannette RL, Fukami T. 2016 Nectar microbes can reduce secondary metabolites in nectar and alter effects on nectar consumption by pollinators. Ecology 97, 1410-1419. (doi:10.1890/15-0858.1)

16. Schaeffer RN, Mei YZ, Andicoechea J, Manson JS, Irwin RE. 2017 Consequences of a nectar yeast for pollinator preference and performance. Funct. Ecol. 31, 613-621. (doi:10.1111/1365-2435.12762)

17. Pozo MI, Bartlewicz J, van Oystaeyen A, Benavente A, van Kemenade G, Wäckers F, Jacquemyn H. 2018 Surviving in the absence of flowers: do nectar yeasts rely on overwintering bumblebee queens to complete their annual life cycle? FEMS Microbiol. Ecol. 94. (doi:10.1093/femsec/fiy196)

18. Koch H, Abrol DP, Li J, Schmid-Hempel P. 2013 Diversity and evolutionary patterns of bacterial gut associates of corbiculate bees. Mol. Ecol. 22, 2028-2044. (doi:10.1111/mec.12209)

19. Graystock P, Rehan SM, McFrederick QS. 2017 Hunting for healthy microbiomes: determining the core microbiomes of Ceratina, Megalopta, and Apis bees and how they associate with microbes in bee collected pollen. Conserv. Genet. 18, 701-711. (doi:10.1007/s10592-017-0937-7)

20. Lachance M-A, Starmer WT, Rosa CA, Bowles JM, Barker JSF, Janzen DH. 2001 Biogeography of the yeasts of ephemeral flowers and their insects. FEMS Yeast Res. 1, 1-8. (doi:10.1111/j.1567-1364.2001.tb00007.x)

21. Lenaerts M et al. 2017 Nectar bacteria affect life history of a generalist aphid parasitoid by

22. Pinheiro J, Bates D, DebRoy S, Sarkar D, R Core Team. 2018 nlme: Linear and Nonlinear

23. R Core Team. 2013 R: A Language and Environment for Statistical Computing. Vienna, Austria: R Foundation for Statistical Computing. See http://www.R-project.org/.

24. Russell AL, Ashman T-L. In press. Associative learning of flowers by generalist bumble ecology of insect-yeast relationships and its relevance to human industry. Proc. R. Soc. B 285, 20172733. (doi:10.1098/rspb.2017.2733) 


\section{Figure legends}

256 Figure 1 Behavioral (A) and gustatory (B) responses of bumble bees to artificial nectar

257 colonized by nectar-inhabiting microbes and the volatile organic compounds they emit.

Table 1 - Volatile organic compounds produced by nectar-inhabiting microorganisms and their respective normalized mean bumble bee electroantennogram (EAG) response \pm standard error $(n=6)$ and corresponding false discovery rate corrected $p$-values.

\begin{tabular}{|c|c|c|c|c|c|}
\hline \multirow[b]{2}{*}{ Class } & \multirow[b]{2}{*}{ Chemical } & \multicolumn{2}{|c|}{$\begin{array}{l}\text { Presence in nectar } \\
\text { headspace }^{\mathrm{a}}\end{array}$} & \multirow{2}{*}{$\begin{array}{l}\text { Normalized } \\
\text { EAG response }^{b} \\
(\% ; n=6 \text { bees })\end{array}$} & \multirow{2}{*}{$\begin{array}{c}\mathrm{P}- \\
\text { value }\end{array}$} \\
\hline & & A. astilbes & M. reukaufii & & \\
\hline \multirow[t]{11}{*}{$1^{\circ}$ Alcohol } & ethanol & ++ & ++++ & $-12 \pm 14$ & 0.72 \\
\hline & 1-propanol & - & ++ & $-2 \pm 4$ & 0.80 \\
\hline & isobutanol & + & +++ & $-8 \pm 6$ & 0.67 \\
\hline & 3-methyl-1-butanol & ++ & ++++ & $-7 \pm 9$ & 0.72 \\
\hline & 3-methyl-3-buten-1-ol & - & + & $-9 \pm 9$ & 0.72 \\
\hline & 4-penten-1-ol & - & + & $-5 \pm 5$ & 0.72 \\
\hline & 1-hexanol & + & + & $66 \pm 42 *$ & 0.047 \\
\hline & 3-ethoxy-1-propanol & - & + & $-4 \pm 14$ & 0.80 \\
\hline & 2-ethyl-1-hexanol & ++ & + & $144 \pm 8 * * *$ & 0.00025 \\
\hline & 2-methyl-1-butanol & ++ & ++++ & $-12 \pm 14$ & 0.72 \\
\hline & 2-phenylethanol & + & +++ & $73 \pm 13 *$ & 0.022 \\
\hline $2^{\circ}$ Alcohol & 2-butanol & - & + & $-5 \pm 8$ & 0.72 \\
\hline Aldehyde & acetaldehyde & + & ++ & $23 \pm 7^{\dagger}$ & 0.07 \\
\hline \multirow[t]{4}{*}{ Ester } & ethyl acetate & - & +++ & $-5 \pm 7$ & 0.72 \\
\hline & 2-methylpropyl acetate & - & + & $20 \pm 7$ & 0.11 \\
\hline & ethyl butyrate & - & + & $-10 \pm 18$ & 0.76 \\
\hline & 3-methylbutyl acetate & - & + & $24 \pm 6 *$ & 0.047 \\
\hline Isoprenoid & isoprene & + & - & $-1 \pm 9$ & 0.93 \\
\hline Ketone & 3-hydroxy-2-butanone & ++ & ++ & $54 \pm 35$ & 0.52 \\
\hline Misc. & 2,5-dimethylfuran & ++ & - & $-5 \pm 15$ & 0.80 \\
\hline
\end{tabular}

$258{ }^{a}$ Relative abundance of volatiles are represented by $+/$ - symbols where - indicates a chemical was

259 not or infrequently detected (one replicate or less) and + symbols correspond to the relative peak

260 areas orders of magnitude in microbial headspace after $96 \mathrm{~h}$ growth in synthetic nectar as

261 reported in [5]. ${ }^{\mathrm{b}}$ Normalized mean response is significantly different from 0 (false discovery rate

$262 \dagger \mathrm{P}<0.1, * \mathrm{P}<0.05, * * \mathrm{P}<0.01, * * * \mathrm{P}<0.001)$. 
bioRxiv preprint doi: https://doi.org/10.1101/549279; this version posted February 20,2019 . The copyright holder for this preprint (which was not certified by peer review) is the author/funder, who has granted bioRxiv a license to display the preprint in perpetuity. It is made available under aCC-BY-NC-ND 4.0 International license.

\section{Figure 1}
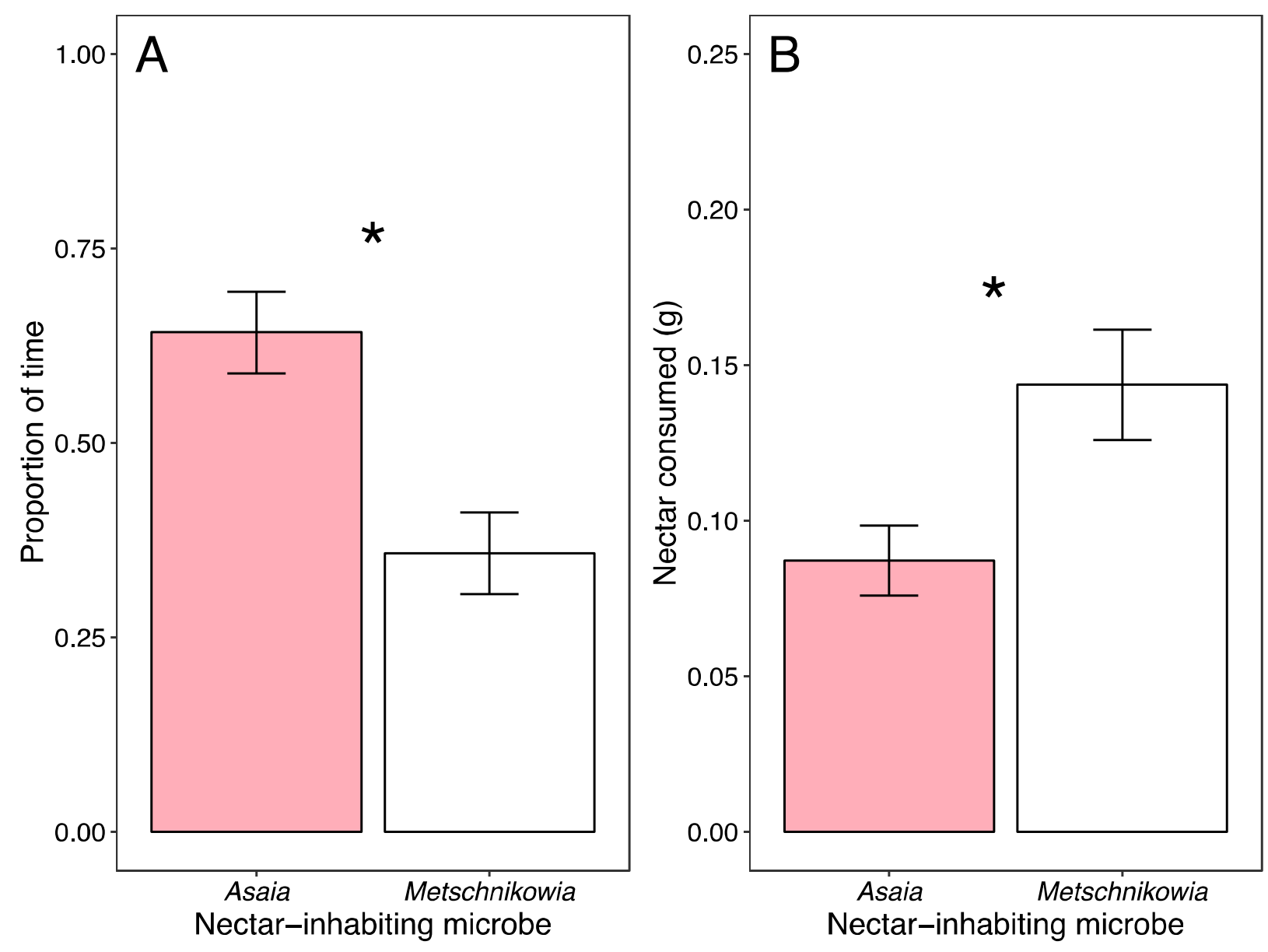